\title{
Exercise Holds Immediate Benefits for Affect and Cognition in Younger and Older Adults
}

\author{
Candice L. Hogan, \\ Department of Psychology, Stanford University \\ Jutta Mata, and \\ Department of Psychology, Stanford University; Center for Adaptive Rationality, Max Planck \\ Institute for Human Development, Berlin, Germany
}

\author{
Laura L. Carstensen \\ Department of Psychology, Stanford University
}

\begin{abstract}
Physical activity is associated with improved affective experience and enhanced cognitive processing. Potential age differences in the degree of benefit, however, are poorly understood because most studies examine either younger or older adults. The present study examined age differences in cognitive performance and affective experience immediately following a single bout of moderate exercise. Participants (144 community members aged 19 to 93 ) were randomly assigned to one of two experimental conditions: (a) exercise (15 min of moderate intensity stationary cycling) or (b) control (15 min completing ratings of neutral IAPS images). Before and after the manipulation, participants completed tests of working memory and momentary affect experience was measured. Results suggest that exercise is associated with increased levels of higharousal positive affect (HAP) and decreased levels of low-arousal positive affect (LAP) relative to control condition. Age moderated the effects of exercise on LAP, such that younger age was associated with a drop in reported LAP postexercise, whereas the effects of exercise on HAP were consistent across age. Exercise also led to faster RTs on a working memory task than the control condition across age. Self-reported negative affect was unchanged. Overall, findings suggest that exercise may hold important benefits for both affective experience and cognitive performance regardless of age.
\end{abstract}

\section{Keywords}

physical activity; emotion; working memory; aging; $n$-back task

\begin{abstract}
Health experts often remark that if exercise came in pill form it would be the most soughtafter drug on the market. For decades, research has frequently identified exercise as an important tool for enhancing a range of physical indices from balance, bone density, strength, and endurance to lipid profiles, blood pressure, and cardiovascular health (Atha, 1981; Bassey \& Ramsdale, 1994; Hickson, Bomze, \& Holloszy, 1977; Judge, Lindsey, Underwood, \& Winsemius, 1993; Thompson et al., 2003). More recent studies have associated exercise with improved brain health (e.g., increased secretion of neuroprotective factors, such as brain-derived neurotrophic factor; Zoladz et al., 2009); and improved
\end{abstract}

\footnotetext{
(C) 2013 American Psychological Association

Correspondence concerning this article should be addressed to Candice L. Hogan, Department of Psychology, Stanford University, 450 Serra Mall, Bldg 420 Jordan Hall, Stanford, CA 94306. cdlowder@stanford.edu.
} 
profiles for markers of cellular aging (e.g., telomere length and autophagy; He et al., 2012; Ludlow et al., 2008).

Though most research focuses on potential benefits to physical health, there is mounting evidence that exercise benefits affective experience and cognitive performance. Researchers report reliable associations in younger adults between exercise and increased positive affect (see Reed \& Ones, 2006 for a meta-analysis) and reductions in negative affect and other depressive symptoms (e.g., Mead et al., 2009 for a meta-analysis). Moderate and vigorous exercise bouts ranging from 5 to $30 \mathrm{~min}$ are associated with improved psychological wellbeing and positive affective responses relative to controls (Barton \& Petty, 2010; Cox, Thomas, Hinton, \& Donahue, 2006; Daley \& Welch, 2004; Hansen, Stevens, \& Coast, 2001). In a review of 25 studies employing the Profile of Mood States in laboratory exercise studies, results indicated that exercise is typically associated with reductions in tension, anger, depression, and confusion (Berger \& Motl, 2000). Mata, Hogan, Joorman, Waugh, and Gotlib (2013) tested whether exercise mitigates consequences of exposure to emotional stressors in individuals recovered from major depressive disorder and healthy control participants. Participants were randomly assigned to an exercise or rest condition before exposure to two sad mood inductions. Recovered depressed participants who had exercised and healthy controls showed no increase in negative affect in response to repeated sad mood inductions, whereas recovered depressed participants who had not exercised reported higher negative affect following the second sad mood induction, suggesting that exercise may serve as a protective factor against exposure to emotional stressors. In addition, studies have found that increased levels of exercise in everyday life produce increased positive affect in healthy college students (Giacobbi, Hausenblas, \& Frye, 2005) and in young adults with major depressive disorder (Mata et al., 2012).

Exercise is also associated with improvements in cognitive performance in younger and older adults. Over the last several decades, numerous studies have tested both the effects of single, acute bouts of exercise and longer term (e.g., 3- or 6-month) interventions. In a review of 43 studies assessing performance on various cognitive tasks following single, acute bouts of exercise, exercise has been linked to improvements in cognitive performance in young adults for tasks ranging from simple reaction time (RT) to response inhibition to creative thinking (Tomporowski, 2003). Hillman, Snook, and Jerome (2003) examined college students' event-related brain potentials and performance on an Eriksen flanker task following $30 \mathrm{~min}$ of treadmill exercise. They found that exercise was related to greater P3 amplitude (an indicator of allocation of cognitive resources), suggesting that acute bouts of cardiovascular exercise may facilitate the allocation of attentional and memory resources and thereby benefit executive functioning (Hillman et al., 2003). A limitation of research testing benefits to cognitive performance following exercise has been the focus on younger adult samples. For example, Chang and colleagues' (2012) meta-analysis identified 79 studies that assessed cognitive performance in association with acute exercise, and of these, only six studies included participants aged 60 years or older, while 42 studies sampled participants aged 20 to 30 years. In contrast, longer-term interventions have focused mainly on older adults. Colcombe and colleagues (2006) randomly assigned sedentary older adults to participate in either vigorous, aerobic exercise training or a stretching control group three times per week for six months. Participants underwent MRI both before and after exercise training. Following the intervention, participants in the aerobic training group showed significant increases in both gray-and white-matter brain regions compared with control participants. More recent evidence suggests that volumetric differences in brain regions may mediate cognitive performance differences, such as task switching (Verstynen et al., 2012). In a review of 18 interventions examining the effects of aerobic training on cognitive function in older adults, Colcombe and Kramer (2003) found a moderate effect size (.48) for 
training benefits to a variety of cognitive processes, especially executive control processes (e.g., working memory, inhibitory processes, multitasking).

All told, evidence for the benefits of exercise on both affective experience and cognitive performance points to exercise as an effective, low-cost intervention for improving both affective and cognitive health. However, there has been some suggestion in the literature that the effects of exercise may be weaker for older than for younger adults. The influence of exercise on affective experience appears somewhat mixed in studies focusing on older adults, with some studies reporting affective benefits from exercise and others reporting reductions in positive affective states following exercise (Arent, Landers, \& Etnier, 2000; Blumenthal et al., 1989; Focht, Knapp, Gavin, Raedeke, \& Hickner, 2007; Focht, Gauvin, \& Rejeski, 2004). In addition, Ruuskanen and Ruoppila (1995) observed that the impact of exercise on well-being was weaker for participants aged 76 and older than for participants aged 65 to 75 years of age. A recent meta-analysis of studies on the effects of physical activity on well-being at advanced ages also concluded that the benefits of exercise are weakened with age, with a gradual decrease in the degree of benefits at older ages (Netz, $\mathrm{Wu}$, Becker, \& Tenenbaum, 2005). However, findings suggesting a diminishing effect of exercise in older adults are difficult to interpret due to a paucity of studies directly comparing effects of the same type and duration of exercise on affective experience and cognitive performance across age groups.

Among studies that have directly compared age groups, results have been mixed. One 4week daily diary study, which assessed emotion and physical activity in younger and older adults, concluded that younger adults reap more emotional benefits from light leisure-time physical activities than older adults (Ready, Marquez, \& Akerstedt, 2009). A study of British citizens aged 18 to 94 tested associations among self-reported walking, age, and cognitive function, and observed a significant interaction of walking and age on simple and choice RT tasks, suggesting that walking may help to attenuate the association between older age and slowed RT (Emery, Huppert, \& Schein, 1995). More recently, Whitbourne, Neupert, and Lachman (2008) observed a relatively stronger reduction in daily perceived memory failures associated with leisure-time physical activity in older versus younger adults. However, though diary studies offer many advantages, the reliance on self-reports about activities in day to day life lack precision, especially regarding dose (i.e., observed differences may derive from different amounts and intensities of exercise). Kamijo and colleagues (2009) compared the effects of light and moderate exercise in 12 older and 12 younger adults on RTs on a test of interference control (i.e., flanker task) and observed marginal differences such that both older and younger adults demonstrated faster RTs following moderate exercise compared with baseline. However, because this study was conducted over three separate sessions at 12-day intervals, it is unclear whether changes in flanker performance were solely attributable to exercise. Thus, conclusions about the effectiveness of exercise-based interventions remain highly tentative. Research utilizing objective measurements of exercise, as well as proximal measures of affective experience and cognitive performance in association with exercise across different age groups, is needed to reconcile mixed evidence.

To recapitulate, the body of research comparing the effects of exercise in younger and older adults is sparse, and existing age-comparative studies have been largely observational, mostly relying on self-report, as well as delayed measurement of affect and cognition, such as single, end-of-day rating of affect, regardless of timing of physical activity. The purpose of the present study is to systematically compare the effects of a single, acute bout of exercise compared with a nonexercise control condition, on both affect and cognitive performance across a broad age range to determine whether and how age may moderate these effects. 


\section{Method}

\section{Participants}

Residents from the community were recruited via advertisements on Internet bulletin boards and university kiosks to participate in a lab-based study on exercise. Once participants contacted research staff to indicate their interest, they were screened for eligibility using the Physical Activity Readiness Questionnaire (PAR-Q) to assess presence of conditions that might make physical activity too risky to participate, such as heart or joint problems (Thomas, Reading, \& Shephard, 1992). Only participants who answered "no" to all questions or received verbal permission from his or her doctor were included in the study. The telephone version of the Mini-Mental State Examination (MMSE) was administered to screen for potential cognitive impairment (Newkirk et al., 2004). Only participants scoring greater than or equal to 23 points on the 26-point scale were enrolled in the study.

Participants aged 19 to 93 years $(N=144)$ completed the study. Participants received $\$ 20$ as compensation. Means $(M)$ and standard deviations $(S D)$ for demographic characteristics and descriptive measures are presented in Table 1.

\section{Measures}

Affect assessment-Ekkekakis and Petruzzello (2002) have recommended the use of measures that capture the affective circumplex, including both high- and low-arousal positive and negative states for studies testing the affective effects of exercise; therefore a modified version of the emotion sampler used by Carstensen and colleagues (2011) and Carstensen, Pasupathi, Mayr, \& Nesselroade (2000) was selected for this study. Thirteen emotion words (angry, anxious/worried, sad, fatigued, bored, quiet, activated, enthusiastic, excited, calm, content, relaxed, happy) representative of the affective circumplex described by Barrett and Russell (1999) were selected for affect assessment. At each assessment, these words appeared sequentially on a computer screen, and participants were asked to indicate the degree to which they were feeling each emotion in that moment on a scale ranging from 1 (Very little or not at all) to 5 (Extremely). Word order was randomized at each assessment for each participant.

This measure was administered twice during the study: (a) At baseline (immediately after completing informed consent and before completing the 2-back task (described in the next section), approximately 5 min before completing the exercise or control condition) and (b) immediately following the experimental or control condition. Composite affect scores were created by averaging across low-arousal positive (LAP) words (calm, content, relaxed; $\alpha \geq$. 72 ) and high-arousal positive (HAP) words (activated, excited, enthusiastic; $\alpha \geq .74$ ). Negative affective states showed very low variability at both measurement points (all medians $=1$, with the exception of fatigue after experimental manipulation, for which median $=2$ ), and were therefore not considered further in the analyses.

Cognitive performance-Working memory was assessed using an $n$-back task. During this task, numbers (0-9) are presented one at a time on a computer screen, and participants are asked to indicate whether each new number appearing on the screen matches the one seen $n$ items previously. In our study, participants completed two blocks of a 1-back version of the task as practice in the baseline assessment before completing four blocks of the 2back version.

After participating in the exercise or control condition, participants completed four additional blocks of the 2-back task. Each block was comprised of 22 trials, and stimuli were presented for $500 \mathrm{~ms}$, followed by presentation of a blank screen for 2,500 ms. Items (numbers 0-9) for all trials were randomly generated, such that $33 \%$ of the items in each 
block were targets and the remaining 67\% were nontargets (Gray, 2001; Huxhold, Li, Schmiedek, \& Lindenberger, 2006; Scheibe \& Blanchard-Fields, 2009). Accuracy and RTs were recorded for each trial.

The first two trials of each 2-back block were, by definition, nontargets and were therefore excluded from analyses. Average accuracy was calculated across the four blocks at baseline and again following experimental condition. Average RTs for correct responses were calculated as the average RT across the four blocks for baseline and following experimental condition. Trials with RTs of less than $100 \mathrm{~ms}$ were treated as inaccurate responses.

Perceived exertion-Perceived physical exertion was assessed during exercise and control conditions using Borg's Rating of Perceived Exertion (RPE) scale, a 15-point scale ranging from 6 to $20(6=$ no exertion at all, $20=$ maximal exertion; Borg, 1970 $)$.

Questionnaires-Participants also completed items assessing demographic characteristics and reported typical weekly physical activity with the Paffenbarger Physical Activity Inventory (PPAI; Paffenbarger, Wing, \& Hyde, 1978).

\section{Procedures}

Participants were assigned by stratified randomization within gender (male or female) and age group (young: 19-39 years, middle age: 40-64 years, older age: 65 years or older) to either the exercise or control condition. Participants were asked not to eat or smoke for two hours prior to their study session, to refrain from exercise on the day of study, and to wear comfortable clothing and shoes to the session.

At the start of the session, participants provided informed consent and were outfitted with a Polar heart rate monitor (Polar Electro, Inc., Oula, Finland). Resting heart rate was recorded after three min of seated rest. Next, participants completed a baseline affect assessment, followed by the 2-back task. After completing these tasks, participants immediately completed either the control or exercise condition.

Experimental condition-Stationary bicycling was used as the mode of exercise because it is suitable for participants of all ages. Participants were asked to pedal at a pace of $50 \mathrm{rpm}$ (visible on the display screen of the bike). A warm-up phase ( $M=4.21 \mathrm{~min}, S D=1.42 \mathrm{~min}$ ) was used to gradually increase workload to raise each participant's heart rate to a moderateintensity exercise level of approximately $50 \%$ of heart rate reserve (HRR; Karvonen, Kentala, \& Mustala, 1957). HRR is preferable to the simpler age-predicted percent heart rate because it takes into account the possible range of an individual's heart rate. Participants exercised at this level for 15 min before completing a 3-min cool-down during which the workload was removed. Every $5 \mathrm{~min}$, participants' heart rate was recorded from the heart rate monitor and they were asked to rate their perceived physical exertion using Borg's RPE scale. Moderate exercise intensity is defined as $40 \%$ to $60 \%$ HRR (American College of Sports Medicine, 2009); average exercise intensity of participants in our study was $47 \%$ HRR $(S D=14.7 \%)$. RPE scale ratings between 11 and 13 represent moderate subjective exercise intensity; exercise participants' average rating in our study was $12.74(S D=1.35)$, indicating that they indeed perceived the bout of exercise as moderate. ${ }^{1}$

Control condition-Participants in the control condition were asked to provide subjective picture quality ratings for 90 neutral International Affective Picture System images (IAPS; Lang, Bradley, \& Cuthbert, 1997). Each image was presented for $10 \mathrm{~s}$, after which participants were given $5 \mathrm{~s}$ to provide quality ratings on a 1 (very poor) to 7 (very high) scale, such that the minimum amount of time to complete this task was 15 min and the 
maximum was $22.5 \mathrm{~min}(M=17.77 \mathrm{~min}, S D=.67 \mathrm{~min})$, which roughly matched the duration of the exercise condition. As in the exercise condition, participants' heart rate and perceived physical exertion were recorded every $5 \mathrm{~min}$. Average rating of perceived exertion for control participants was $7.62(S D=1.81)$, indicating that this task was not perceived as physically effortful.

After completing either the exercise or control condition, participants immediately completed the affect assessment, followed by the 2-back task. Participants completed the PPAI (Paffenbarger, Wing, \& Hyde, 1978) and demographic questions and were thanked and debriefed before leaving the session.

\section{Data Analysis}

Independent samples $t$-tests and chi-square tests of contingency were used to test whether baseline differences existed between exercise and control participants' demographic characteristics, including age, body mass index (BMI), physical activity levels, gender, education, or ethnicity. Independent samples $t$-tests were also used to test for baseline differences between exercise and control participants in all dependent measures (HAP affect, LAP affect, 2-back accuracy, and 2-back RT). Both accuracy and RT data from the 2back task were skewed and therefore log-transformed to approximate normal distribution prior to analyses. Data from two participants (one from the exercise condition and one from the control condition) were excluded from 2-back analyses because their initial scores were $\geq 3 S D$ s lower than those of other participants. Change scores for HAP affect, LAP affect, 2back accuracy, and 2-back RT were computed as the standardized residuals obtained by regressing postmanipulation scores on baseline scores. To test whether age moderated the effects of exercise on HAP affect, we regressed change in HAP affect on condition, age, and the interaction of condition and age. We repeated these procedures for change in LAP affect, change in 2-back accuracy, and change in 2-back RT. Age was centered prior to analyses to facilitate interpretation of interaction terms and to reduce the possibility of multicollinearity in regression equations as recommended by Cohen, Cohen, West, and Aiken (2003). When significant interactions were observed, we tested the simple slopes for the association between condition and age (Aiken \& West, 1991). Effect sizes are presented for independent samples $t$-tests, Cohen's $d$, calculated as $\left(M_{1}-M_{2}\right) / S D_{\text {pooled }}$, and chi-square tests, phi $(\varphi)$, calculated as $\left(\mathrm{X}^{2} / N\right)^{1 / 2}$.

\section{Results}

\section{Demographic Characteristics of Participants}

There were no baseline differences between exercise and control participants in age, $t(142)$ $=0.16, p=.876, d=0.03$, BMI, $t(142)=0.36, p=.722, d=0.06$, physical activity levels, $t(142)=1.03, p=.304, d=0.17$; gender, $\mathrm{X}^{2}(1)=.11, p=.737, \varphi=-0.03$; education, $\mathrm{X}^{2}(6)=$ $5.50, p=.481, \varphi=.20$; or ethnicity, $\mathrm{X}^{2}(5)=8.52, p=.130, \varphi=.24$.

\section{Effects of Experimental Condition and Age on Affective Experience}

Means and standard deviations for baseline and follow-up measures of affect and cognitive performance are presented in Table 2. There were no significant differences between

\footnotetext{
${ }^{1}$ To test whether exercise intensity influenced affect in the current study, we regressed change in HAP affect and LAP affect on HRR percentage and RPE for exercise participants. There was no association between HRR $\%$ and change in HAP affect $\left(b^{*}=.08, p=\right.$ $480)$ or LAP affect $\left(b^{*}=.08, p=.533\right)$. There was also no relation between RPE and change in HAP affect $\left(b^{*}=-.07, p=.569\right)$. We observed an effect of RPE on change in LAP affect such that higher perceived exertion was associated with less LAP affect following exercise $\left(b^{*}=-.31, p=.009\right)$. We next included RPE as a covariate in our model predicting change in LAP by condition, age, and the interaction of condition and age; however, RPE was not a significant predictor of change in LAP within this model $\left(b^{*}=-.20, p=\right.$. 207) and inclusion of RPE did not significantly improve the fit of the model, $P(1,139)=1.61, p=.207$.
} 
conditions for baseline HAP states, $t(142)=-0.21, p=.834, d=-0.04$, or LAP states, $t(142)=0.07, p=.944, d=0.01$. We regressed baseline affect composite scores on age to test for initial differences of age in affect ratings. Results indicated that age did not significantly predict baseline $\operatorname{HAP}\left(b^{*}=0.03, p=.750\right)$ or $\operatorname{LAP}\left(b^{*}=-0.05, p=.546\right)$.

In terms of HAP, we observed an effect of condition such that exercise participants reported a greater increase in HAP than did controls $\left(b^{*}=0.34, p<.001\right)$. We did not observe an effect of age on change in HAP $\left(b^{*}=-0.26, p=.278\right)$. We observed an Age $\times$ Condition interaction $\left(b^{*}=0.45, p=.046\right) ; \Delta R^{*}=.02$. To understand this interaction, we tested the statistical significance of the slopes of the simple regression lines representing relations between age and HAP using dummy coding for condition. There was a significant positive slope for control condition $\left(b^{*}=0.36, p=.002\right)$ but not for exercise $\left(b^{*}=0.05, p=.632\right)$, indicating that the effect of the control condition on HAP was moderated by age, but the effect of exercise on HAP was relatively equal across the age range sampled (see Figure 1).

Testing the effect on LAP, we observed a marginal effect of condition such that participants who exercised reported greater decrease in LAP than those in the control condition $\left(b^{*}=-\right.$. $14, p=.075)$. Interestingly, we also observed a significant interaction of age and condition $\left(b^{*}=0.55 p=.028\right) ; \Delta R^{2}$ associated with the interaction term $=.03$. Age predicted greater increase in LAP $\left(b^{*}=0.74, p=.004\right)$. To understand the interaction, we tested the statistical significance of the slopes of the simple regression lines representing relations between age and LAP using dummy coding for condition. There was a significant positive slope for exercise $\left(\mathrm{b}^{*}=0.38, p=.001\right)$ but not for control $\left(b^{*}=0.02, p=.863\right)$, indicating that the effect of the exercise condition on LAP was moderated by age, but the change in LAP associated with the control condition was relatively equal across the age range sampled (see Figure 1).

\section{Effects of Experimental Condition and Age on Cognitive Performance}

There was no baseline difference between conditions for 2-back accuracy, $t(140)=-0.28, p$ $=.783, d=-0.05$, but there was a trend for a difference in RT such that participants in the exercise condition were initially slower than participants in the control condition, $t(140)=$ $1.74, p=.083, d=0.29$. To test for initial differences of age in cognitive performance, we regressed baseline 2-back accuracy and RT on age. As expected, age was significantly related to accuracy on the $n$-back task $\left(b^{*}=-0.28, p<.001\right)$ and $\mathrm{RT}\left(b^{*}=.38, p<.001\right)$, such that older age compared with younger age predicted lower accuracy and slower responding, respectively. We did not observe a significant effect of age on change in accuracy $\left(b^{*}=-0.39, p=.135\right)$, of condition on change in accuracy $\left(b^{*}=-0.09, p=.274\right)$, or a significant interaction of age and condition on 2-back accuracy $\left(b^{*}=0.23, p=.377\right.$; see Figure 2). Regarding change in 2-back RT, we observed an effect of condition such that exercise was associated with a greater reduction in RT relative to the control condition $\left(b^{*}=\right.$ $0.20, p=.014)$. Age was not associated with change in 2-back RT $\left(b^{*}=0.10, p=.692\right)$ independent of condition, and we did not observe a significant interaction of condition and age on 2-back RT ( $\left.b^{*}=0.22, p=.379\right)$, indicating that the effect of exercise on reduction in RT was relatively consistent across the age range sampled (see Figure 2).

\section{Relations Between Experimental Condition, Affect, and Cognitive Performance}

Using a series of linear regression models, we tested whether the magnitude of change in HAP or LAP affect was associated with change in 2-back accuracy or RT as a function of experimental condition. We did not observe any significant associations (all $p s>$.442) for change in HAP affect on change in 2-back accuracy or RT, or any interactions of condition and change in HAP affect on change in these measures of cognitive performance. We also did not observe any significant associations (all $p s>$. 493) for change in LAP affect on 
change in 2-back accuracy or RT, or any interactions of condition and change in LAP affect on change in these measures of cognitive performance. Thus, data from the present study suggest that magnitude of change in HAP or LAP affect is not related to change in cognitive performance as a function of condition. ${ }^{2}$

\section{Discussion}

A growing literature demonstrates that exercise benefits both affective experience and cognitive performance. The purpose of this study was to test whether age may moderate these effects. Our results suggest that a single bout of exercise appears to have comparable and positive effects on both affective experience and cognitive performance, independent of participants' age. We observed that a single bout of moderate exercise was associated with increased levels of HAP affect and that the effect was consistent across the age range sampled. Results were slightly different for LAP affect. Younger age was associated with a drop in reported LAP affect, whereas older age was associated with the maintenance, and even slight increase in LAP, postexercise. Regarding effects of acute exercise on cognitive performance, we found that, independent of age, exercise resulted in significant improvement in 2-back RT compared with control participants.

In the current study, age was found to moderate the experience of calm states following exercise, such that younger adults reported decreased LAP affect, but older adults appeared to maintain LAP even after exercise. This finding highlights the importance of measuring the impact of exercise on both valence and arousal components of affect. Although many studies in younger adults observe shifts in HAP states (e.g., feelings of energy), the relationship between exercise and LAP states is less clear. To promote more precise understanding of the relation between affect and exercise, the current study followed recommendations from Ekkekakis and Petruzello (2002) by using an affective measure aimed at capturing the full affective circumplex. These methods allowed us to assess the influence of exercise on both high- and low-arousal affective states. In addition, recent findings suggest that, as people age, they may come to experience, value, and seek out lowarousal positive states, such as feeling calm, to a greater degree than younger adults (Scheibe, English, Tsai, \& Carstensen, 2013). Therefore, as demonstrated by the present findings, it may be particularly important to consider both low- and high-arousal affective changes in response to exercise to best characterize affective effects of exercise across the life span.

In the present study, we observed that the effects of exercise compared with a control condition on cognitive performance appeared relatively stable across the age range sampled. Specifically, we found that, independent of age, exercise resulted in significant improvement in 2-back RT compared with control condition, and we did not observe an effect of exercise or control condition on change in 2-back accuracy. Although results from the present study suggest that changes in affect and cognitive performance observed in the exercise condition occur independently, future research should replicate these results and further investigate potential underlying mechanisms to better understand how exercise confers psychological benefits.

\footnotetext{
${ }^{2}$ At the suggestion of an anonymous reviewer, we also examined a potential speed-accuracy trade-off that may have differed between the age groups. We regressed change in 2-back RT, age, and the interaction of 2-back RT and age on change in 2-back accuracy for participants randomly assigned to the exercise condition. We did not observe a significant association between change in 2-back RT or change in 2-back accuracy $\left(b^{*}=0.16, p=.235\right)$ or an interaction between change in 2-back RT and age on 2-back accuracy $\left(b^{*}=\right.$ $0.005, p=.452$ ); however, we agree with the reviewer that future research should assess the presence of speed-accuracy trade-offs in studies examining the effects of exercise on cognitive performance.
} 
It is important to acknowledge three limitations of the current research. First, this study tested effects of one acute bout of exercise. It remains to be shown whether and how repeated exercise participation may differentially benefit people of different ages. Although age may not moderate the effects of a single bout of exercise on cognitive performance, further research is needed to test whether longer term effects are comparable across the adult age range. Second, all study participants were in relatively good health (e.g., did not report conditions that would make exercising potentially dangerous, such as a heart condition); thus, it remains unclear whether these results might generalize to those in poorer health. A third limitation concerns the temporal separation between baseline and follow-up measures of affect and cognitive performance for the exercise participants compared with control participants. Despite the same overall session duration, the timing of the affect and cognitive performance measures postexercise/postfiller task were not exactly aligned between the exercise and no-exercise conditions: Due to need for a warm-up phase in the exercise condition, participants in the exercise condition started these measurements about 4 min and $24 \mathrm{~s}$ later than in the control condition, which—albeit unlikely—could have potentially affected our findings.

Findings from the current study help to characterize the nature of the effects of exercise on affective experience and cognitive performance across the adult age range. Under controlled, experimental conditions, individuals across the adult age range experienced comparable benefits. The relatively large sample of individuals drawn from the community and ranging in age from 19 to 93 represents an additional strength of this study and may enhance the generalizability of our findings. Future research is needed to further clarify how these findings may map on to exercise, affect, and cognition in daily life. The findings add to a growing body of research pointing to the importance of exercise for both physical and psychological health by suggesting that, even as individuals age, exercise remains an important contributor to psychological health, including affective experience and cognitive performance.

\section{Acknowledgments}

Research reported in this publication was supported by the National Institute on Aging of the National Institutes of Health under Award Number R37-AG008816 to Laura L. Carstensen. The content is solely the responsibility of the authors and does not necessarily represent the official views of the National Institutes of Health.

\section{References}

Aiken, LS.; West, SG. Multiple regression: Testing and interpreting interactions. Newbury Park, CA: Sage; 1991.

American College of Sports Medicine. General principles of exercise prescription. In: Thompson, WR.; Gordon, NF.; Pescatello, LS., editors. ACSM's guidelines for exercise testing and prescription. 8. New York, NY: Lippincott Williams \& Wilkins; 2009. p. 152-182.

Arent SM, Landers DM, Etnier JL. The effects of exercise on mood in older adults: A meta-analytic review. Journal of Aging and Physical Activity. 2000; 8:407-430.

Atha J. Strengthening muscle. Exercise Sport Science Review. 1981; 9:1-78.

Barrett LF, Russell JA. Structure of current affect. Current Directions in Psychological Science. 1999; 8:10-14.

Barton J, Petty J. What is the best dose of nature and green exercise for improving mental health? A multi-study analysis. Environmental Science \& Technology. 2010; 44:3947-3955.10.1021/ es903183r [PubMed: 20337470]

Bassey EJ, Ramsdale SJ. Increase in femoral bone density in young women following high-impact exercise. Osteoporosis International. 1994; 4:72-75. [PubMed: 8003843] 
Berger BG, Motl RW. Exercise and mood: A selective review and synthesis of research employing the profile of mood states. Journal of Applied Sport Psychology. 2000; 12:69-

92.10.1080/10413200008404214

Blumenthal JA, Emery CF, Madden DJ, George LK, Coleman RE, Riddle MW, Williams RS. Cardiovascular and behavioral effects of aerobic exercise training in healthy older men and women. Journal of Gerontology. 1989; 44:M147-M157. [PubMed: 2768768]

Borg G. Perceived exertion as an indicator of somatic stress. Scandinavian Journal of Rehabilitation Medicine. 1970; 2:92-98. [PubMed: 5523831]

Carstensen LL, Pasupathi M, Mayr U, Nesselroade J. Emotional experience in everyday life across the adult life span. Journal of Personality and Social Psychology. 2000; 79:644-655. doi:10.1037// O022-3514.79.4.64. [PubMed: 11045744]

Carstensen LL, Turan B, Scheibe S, Ram N, Ersner-Hershfield H, Samanez-Larkin GR, Nesselroade JR. Emotional experience improves with age: Evidence based on over 10 years of experience sampling. Psychology and Aging. 2011; 26:21-33.10.1037/a0021285 [PubMed: 20973600]

Chang YK, Labban JD, Gapin JI, Etnier JL. The effects of acute exercise on cognitive performance: A meta-analysis. Brain Research. 2012; 1453:87-101.10.1016/j.brainres.2012.02.068 [PubMed: 22480735]

Cohen, J.; Cohen, P.; West, SG.; Aiken, LS. Applied multiple regression/correlation analysis for the behavioral sciences. 3. Mahwah, NJ: Erlbaum; 2003.

Colcombe SJ, Erickson KI, Scalf PE, Kim JS, Prakash R, McAuley E, Kramer AF. Aerobic exercise training increases brain volume in aging humans. The Journals of Gerontology: Series A Biological Sciences and Medical Sciences. 2006; 61:1166-1170.10.1093/gerona/58.2.M176

Colcombe SJ, Kramer AF. Fitness effects on the cognitive function of older adults: A meta-analytic study. Psychological Science. 2003; 14:125-130.10.1111/1467-9280.t01-1-01430 [PubMed: 12661673]

Cox RH, Thomas TR, Hinton PS, Donahue OM. Effects of acute $60 \%$ and $80 \%$ VO2max bouts of aerobic exercise on state anxiety of women of different age groups across time. Research Quarterly for Exercise and Sport. 2004; 75:165-175. [PubMed: 15209335]

Daley AJ, Welch A. The effects of 15 min and 30 min of exercise on affective responses both during and after exercise. Journal of Sports Sciences. 2004; 22:621628.10.1080/02640410310001655778 [PubMed: 15370492]

Ekkekakis P, Petruzzello SJ. Analysis of the affect measurement conundrum in exercise psychology: IV. A conceptual case for the affect circumplex. Psychology of Sport and Exercise. 2002; 3:3563.10.1016/S1469-0292(01)00028-0

Emery CF, Huppert FA, Schein RL. Relationships among age, exercise, health, and cognitive function in a British sample. The Gerontologist. 1995; 35:378-385.10.1093/geront/35.3.378 [PubMed: 7622090]

Focht BC, Gauvin L, Rejeski WJ. The contribution of daily experiences and acute exercise to fluctuations in daily feeling states among older, obese adults with knee osteoarthritis. Journal of Behavioral Medicine. 2004; 27:101-121.10.1023/B:JOBM.0000019847.80315.4d [PubMed: 15171102]

Focht BC, Knapp DJ, Gavin TP, Raedeke TD, Hickner RC. Affective and self-efficacy responses to acute aerobic exercise in sedentary older and younger adults. Journal of Aging and Physical Activity. 2007; 15:123-138. [PubMed: 17556780]

Giacobbi PR, Hausenblas HA, Frye N. A naturalistic assessment of the relationship between personality, daily life events, leisure-time exercise, and mood. Psychology of Sport and Exercise. 2005; 6:67-81.10.1016/J.Psychsport.2004.10.009

Gray JR. Emotional modulation of cognitive control: Approach-withdrawal states double dissociate spatial from verbal two-back task performance. Journal of Experimental Psychology: General. 2001; 130:436-452.10.1037/0096-3445.130.3.436 [PubMed: 11561919]

Hansen CJ, Stevens LC, Coast JR. Exercise duration and mood state: How much is enough to feel better? Health Psychology. 2001; 20:267-275.10.1037/0278-6133.20.4.267 [PubMed: 11515738] 
He C, Bassik MC, Moresi V, Sun K, Wei Y, Zou Z, Levine B. Exercise-induced BCL2-regulated autophagy is required for muscle glucose homeostasis. Nature. 2012; 481:511-515.10.1038/ nature10758 [PubMed: 22258505]

Hickson RC, Bomze HA, Holloszy JO. Linear increase in aerobic power induced by strenuous program of endurance exercise. Journal of Applied Physiology. 1977; 42:372-376. [PubMed: 838658]

Hillman CH, Snook EM, Jerome GJ. Acute cardiovascular exercise and executive control function. International Journal of Psychophysiology. 2003; 48:307-314.10.1016/S0167-8760(03)00080-1 [PubMed: 12798990]

Huxhold O, Li SC, Schmiedek F, Lindenberger U. Dual-tasking postural control: Aging and the effects of cognitive demand in conjunction with focus of attention. Brain Research Bulletin. 2006; 69:294-305.10.1016/j.brainresbull.2006.01.002 [PubMed: 16564425]

Judge JO, Lindsey C, Underwood M, Winsemius D. Balance improvement in older women: Effects of exercise training. Physical Therapy. 1993; 73:254-262. [PubMed: 8456144]

Kamijo K, Hayashi Y, Sakai T, Yahiro T, Tanaka K, Nishihira Y. Acute effects of aerobic exercise on cognitive function in older adults. The Journals of Gerontology Series B: Psychological Sciences and Social Sciences. 2009; 64:356-363.10.1093/geronb/gbp030

Karvonen MJ, Kentala E, Mustala O. The effects of training on the heart rate: A longitudinal study. Annales Medicinae Experimentalis et Biologiae Fenniae. 1957; 35:307-315. [PubMed: 13470504]

Lang, PJ.; Bradley, MM.; Cuthbert, BN. International affective picture system (IAPS): Technical manual and affective ratings. Gainsville, FL: The Center for Research in Psychophysiology, University of Florida; 1999.

Ludlow AT, Zimmerman JB, Witkowski S, Hearn JW, Hatfield BD, Roth SM. Relationship between physical activity level, telomere length, and telomerase activity. Medicine and Science in Sports and Exercise. 2008; 40:1764-1771. doi:10.1249/MSS.0b013e 31817c92aa. [PubMed: 18799986]

Mata J, Hogan CL, Joorman J, Waugh CE, Gotlib IH. Acute exercise attenuates negative affect following repeated sad mood inductions in persons who have recovered from depression. Journal of Abnormal Psychology. 2013; 122:45-50.10.1037/a0029881 [PubMed: 22985013]

Mata J, Thompson RJ, Jaeggi S, Buschkuehl M, Jonides J, Gotlib IH. Walk on the bright side: Physical activity and affect in major depressive disorder. Journal of Abnormal Psychology. 2012; 121:297308.10.1037/a0023533 [PubMed: 21553939]

Mead GE, Morley W, Campbell P, Greig CA, McMurdo M, Lawlor DA. Exercise for depression. Cochrane Database of Systematic Reviews. 2009:3.10.1002/14651858

Netz Y, Wu MJ, Becker BJ, Tenenbaum G. Physical activity and psychological well-being in advanced age: A meta-analysis of intervention studies. Psychology and Aging. 2005; 20:272284.10.1037/0882-7974.20.2.272 [PubMed: 16029091]

Newkirk LA, Kim JM, Thompson JM, Tinklenberg JR, Yesavage JA, Taylor JL. Validation of a 26point telephone version of the Mini-Mental State Examination. Journal of Geriatric Psychiatry and Neurology. 2004; 17:81-87.10.1177/0891988704264534 [PubMed: 15157348]

Paffenbarger RS, Wing AL, Hyde RT. Physical activity as an index of heart attack risk in college alumni. American Journal of Epidemiology. 1978; 108:161-175. [PubMed: 707484]

Ready RE, Marquez DX, Akerstedt A. Emotion in younger and older adults: Retrospective and prospective associations with sleep and physical activity. Experimental Aging Research. 2009; 35:348-368.10.1080/03610730902922184 [PubMed: 19449246]

Reed J, Ones DS. The effect of acute aerobic exercise on positive activated affect: A meta-analysis. Psychology of Sport and Exercise. 2006; 7:477-514.10.1016/j.psychsport.2005.11.003

Ruuskanen JM, Ruoppila I. Physical activity and psychological well-being among people aged 65 to 84 years. Age and Ageing. 1995; 24:292-296.10.1093/ageing/24.4.292 [PubMed: 7484485]

Scheibe S, Blanchard-Fields F. Effects of regulating emotions on cognitive performance: What is costly for young adults is not so costly for older adults. Psychology and Aging. 2009; 24:217223.10.1037/a0013807 [PubMed: 19290754]

Scheibe S, English T, Tsai JL, Carstensen LL. Striving to feel good: Ideal affect, actual affect, and their correspondence across adulthood. Psychology and Aging. 2013; 28:160-171.10.1037/ a0030561 [PubMed: 23106153] 
Thomas S, Reading J, Shephard RJ. Revision of the physical activity readiness questionnaire (PAR-Q). Canadian Journal of Sport Sciences. 1992; 17:338-345. [PubMed: 1330274]

Thompson PD, Buchner D, Pina IL, Balady GJ, Williams MA, Marcus BH, Wenger NK. Exercise and physical activity in the prevention and treatment of atherosclerotic cardiovascular disease: A statement from the Council on Clinical Cardiology (Subcommittee on Exercise, Rehabilitation, and Prevention) and the Council on Nutrition, Physical Activity, and Metabolism (Subcommittee on Physical Activity). Circulation. 2003; 107:3109-3116. doi:10.1161/01.CIR.0000075572 . 40158.77. [PubMed: 12821592]

Tomporowski PD. Effects of acute bouts of exercise on cognition. Acta Psychologica. 2003; 112:297324.10.1016/S0001-6918(02)00134-8 [PubMed: 12595152]

Verstynen TD, Lynch B, Miller DL, Voss MW, Prakash RS, Chaddock L, Erickson KI. Caudate nucleus volume mediates the link between cardiorespiratory fitness and cognitive flexibility in older adults. Journal of Aging Research. 2012; 2012:1-11.10.1155/2012/939285

Whitbourne SB, Neupert SD, Lachman ME. Daily physical activity: Relation to everyday memory in adulthood. Journal of Applied Gerontology. 2008; 27:331-349.10.1177/0733464807312175

Zoladz JA, Pilc A, Majerczak J, Grandys M, Zapart-Bukowska J, Duda K. Endurance training increases plasma brain-derived neurotrophic factor concentration in young healthy men. Journal of Physiology and Pharmacology. 2008; 59:119-132. [PubMed: 19258661] 

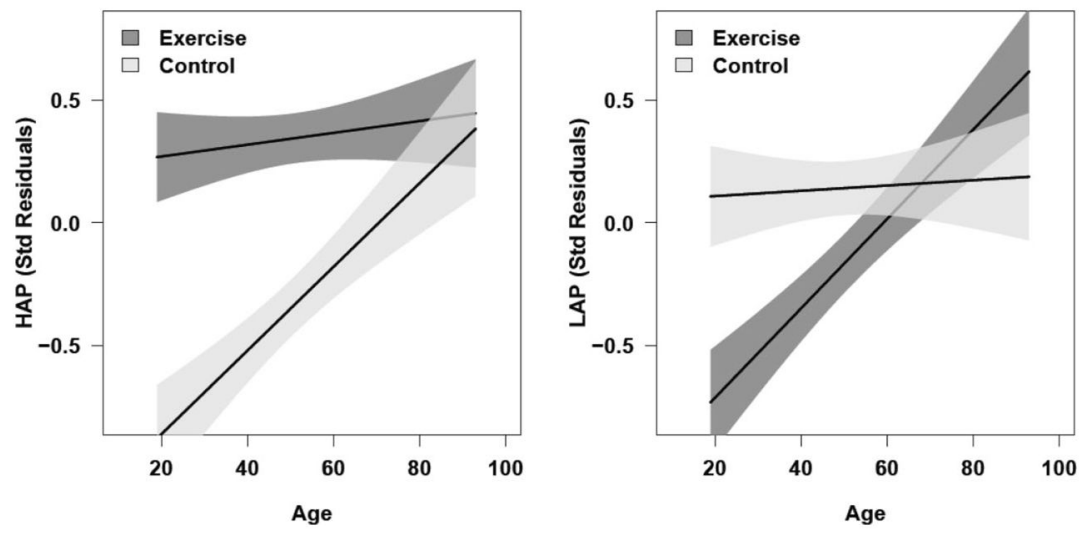

Figure 1.

Effects of experimental condition and age on HAP and LAP affect. Note. Shading represents \pm 1 SEM. High-arousal positive (HAP) affect and low-arousal positive (LAP) affect change scores are presented as standardized residuals obtained by regressing postmanipulation scores on baseline scores. 

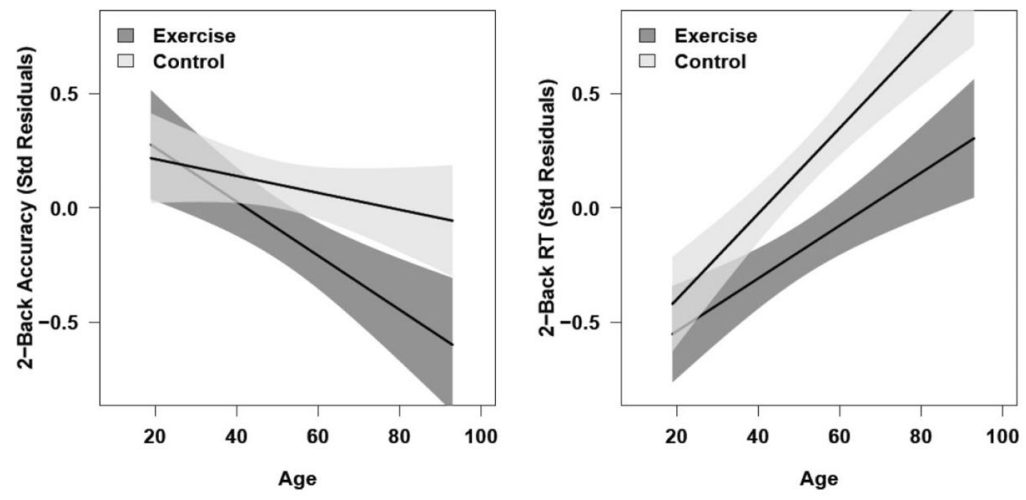

Figure 2.

Effects of condition and age on 2-back accuracy and reaction time. Note. Shading represents \pm 1 SEM. 2-back accuracy and RT change scores are presented as standardized residuals obtained by regressing postmanipulation scores on baseline scores. Negative scores represent faster RT. 


\section{Table 1}

Demographic Characteristics of Participants, Separated by Exercise and Control Condition

\begin{tabular}{lcc}
\hline Variable & Exercise; $\boldsymbol{n}=\mathbf{7 1}$ & Control; $\boldsymbol{n}=\mathbf{7 3}$ \\
\hline Age (years); $M(S D)$ & $51.34(21.74)$ & $50.79(19.96)$ \\
$\mathrm{Q}_{1}$ & $21-29$ & $19-28$ \\
$\mathrm{Q}_{2}$ & $30-49$ & $29-53$ \\
$\mathrm{Q}_{3}$ & $50-72$ & $54-69$ \\
$\mathrm{Q}_{4}$ & $73-93$ & $70-87$ \\
Gender $\%$ male) & 47.9 & 50.7 \\
Ethnicity (\% European American) & 76.1 & 69.9 \\
Education (\% college graduates) & 62 & 67.1 \\
BMI; $M(S D)$ & $27.06(6.19)$ & $26.71(5.41)$ \\
Physical activity (total kcal/wk); $M(S D)$ & $2857.44(2804.96)$ & $2441.88(1968.28)$ \\
\hline
\end{tabular}

Note. Q1 = first quartile; Q2 = second quartile; Q3 = third quartile; Q4 = fourth quartile; BMI = body mass index; physical activity = Paffenbarger Physical Activity Questionnaire. 
Table 2

Descriptive Statistics for Baseline and Follow-Up Affect and Cognitive Performance Variables

\begin{tabular}{lccc}
\hline Variable & Exercise; $\boldsymbol{n}=\mathbf{7 1}$ & Control; $\boldsymbol{n}=\mathbf{7 3}$ & Full Sample; $\boldsymbol{n}=\mathbf{1 4 4}$ \\
\hline Baseline HAP; $M(S D)$ & $2.67(.91)$ & $2.70(.92)$ & $2.68(.91)$ \\
Follow-up HAP; $M(S D)$ & $3.19(.85)$ & $2.70(.97)$ & $2.94(.94)$ \\
Baseline LAP; $M(S D)$ & $3.54(.85)$ & $3.53(.92)$ & $3.53(.88)$ \\
Follow-up LAP; $M(S D)$ & $3.32(.88)$ & $3.52(.76)$ & $3.42(.82)$ \\
Baseline 2-back accuracy; $M(S D)$ & $.88(.08)$ & $.88(.08)$ & $.88(.08)$ \\
Follow-up 2-back accuracy; $M(S D)$ & $.90(.09)$ & $.91(.08)$ & $.90(.08)$ \\
Baseline 2-back RT $(\mathrm{ms}) ; M(S D)$ & $1029.54(281.15)$ & $947.93(304.56)$ & $988(295.09)$ \\
Follow-up 2-back RT $(\mathrm{ms}) ; M(S D)$ & $949.11(279)$ & $944.27(333.62)$ & $946(306.88)$ \\
\hline
\end{tabular}

Note. HAP = high-arousal positive affect LAP = low-arousal positive affect. Scores were transformed prior to analyses (see Data Analysis section); RT = reaction time. 